\title{
A technique for the study of steady-state short term memory
}

\author{
LEONARD KATZ ${ }^{1}$ \\ UNIVERSITY OF CONNECTICUT
}

Steady-state STM was studied by a method in which $S$ was required to keep track of the randomly changing response member of each of 5 stimulus words. On each of $220 \mathrm{con}$ secutive presentations, $S$ had to recall the response last paired with a given stimulus and then had to learn a (possily) new response to the same stimulus. A measure of S's STM was his proportion of correct recalls as a function of the number of items intervening between successive appearances of a given item. Results suggest that the method gives a stable measure of STM. Specifically, proactive effects appeared to be constant throughout the sequence of presentations.

Recently, several investigators have studied shortterm memory (STM) by means of steady-state procedures, i.e., procedures in which $S$ is repeatedly required both to learn new items and to recall old items where both tasks are mixed in a long sequence (e.g., Shepard \& Teghtsoonian, 1961; Hinrichs, 1966). This paper presents a new steady-state method together with data which suggest its validity. Steady-state techniques have many advantages over methods in which $S$ is presented with short sequences of items to remember which are tested for recall only at the end of a given sequence (e.g., Peterson \& Peterson, 1959; Waugh \& Norman, 1965). The steady-state techniques are more efficient in collecting data, are free of primacy effects, and test STM in a relatively more stable context than the shortsequence methods.

The method presented in this paper permits any number of combinations of stimuli, responses, and presentations to be used. In addition to the advantages described above, of steady-state procedures, it has, in paradigm, important similarities to both the anticipation method of paired-associates learning and probability learning. Therefore, validation of the present method would aid in placing the study of STM in a wider context.

In outline, the method is as follows. A test item (a stimulus word) is presented to $\mathrm{S}$ who attempts to recall the response member previously associated with it. Then, the test item is replaced by a reinforced item which is the same stimulus word with a response member added. The response member may or may not be the same response which was previously paired with that stimulus word. Thus, $S$ receives no feedback as to whether or not he correctly recalled the response previously associated with that stimulus word. Instead, he must try to learn a (possibly) different response to the same stimulus. The next presentation begins with the appearance of a (possibly) different test item. Again,
$\mathrm{S}$ attempts to recall the response last associated with that stimulus word. Then, that stimulus word is reinforced with a response that may be different from the response last associated with that stimulus word. Test items alternate with reinforced items in a long sequence of alternations (for example, 200 presentations). Each of a set of stimulus words appears several times in the sequence. The main variable of interest is S's frequency of correct recalls as a function of the number of reinforcements and tests intervening between successive appearances of a given item; this number is defined as lag. Note that each presentation contributes two intervening items, one a test and the other a reinforcement. Method

Five stimuli, the letter-rows BBBB, DDDD, GGGG, TTTT, VVVV, were used in two sequences of 220 presentations each. Each letter-row was assigned one of four responses for each reinforced presentation, the responses being the numbers 1, 2, 3, 4. For each sequence, the first 20 presentations were dummy presentations. In the remaining 200 presentations, each of the 5 letter-rows appeared in 40 presentations and each of the 4 response members was randomly paired 10 times with each letter-row. A reinforcement consisted of presenting a letter-row with one of the four positions enclosed in parentheses, e.g., B B (B) B, the reinforced item "B-3." A test consisted of presenting the letter-row without parentheses, e.g., B B B B. Tests and reinforcement appeared successively in a single window of a Stowe memory drum. Each item remained in the window for $3 \mathrm{sec}$. In between items, a shutter closed for 1 sec. S responded on reinforcements by saying aloud the letter and reinforced position (e.g., "B-3') and by saying aloud on tests the letter and the response he believed to be correct. $S$ was instructed to guess if he did not know the correctanswer. Before the sequence began, $\mathrm{E}$ gave $\mathrm{S}$ a starting-response for $\mathrm{S}$ to make to the initial appearance of each test stimulus.

Each sequence was constructed under the restriction that approximately equal numbers of each lag $0,2,4, \ldots$, 16 occur for each item. ${ }^{2} \mathrm{~S}$ was instructed that the probability of the same item appearing on successive presentations (i.e., a lag of zero) was $1 / 9$ and that this was equal to the probability of any other lag $2,4,6, \ldots$, 16 occurring. Also, $\mathrm{S}$ was told not to rehearse items after they had been presented. By these instructions, it was intended that $S$ pay close attention to each test or reinforced item as it appeared and that $S$ avoid grouping items, looking for "meaningful" subsequences, trying to find consistant differences among items, etc. 
Each of six Ss was given one of two sequences on each of five consecutive days. For three Ss, the order of sequences was $\mathrm{ABABA}$ and for the other three $\mathrm{Ss}$, $\mathrm{BABAB}$. Ss were junior and senior women enrolled at the University of Connecticut. Each was paid $\$ 10$ for participating.

\section{Results and Discussion}

Of major importance is a check of the equilibrium properties of the method. Previous investigators (Shepard \& Teghtsoonian, 1961; Hinrichs, 1966) found that pro-active interference did not increase steadily as the number of presentations increased. The present data support the suggestion that proactive effects are not large and, more importantly, do not operate differentially over the experimental session. Table 1 presents the mean proportion of correct responses in successive blocks of 25 presentations in each of 5 successive days. An analysis of variance was performed on the number of correct responses in successive blocks of 25 presentations in each day for each $\mathrm{S}$. The effect of Blocks was not significant, and, in fact, its mean square was small $(F=1.16, d f=7 / 35)$. Neither was the Blocks by Days interaction significant $(F=1.00, \mathrm{df}=28$ / 140). However, the effect of Days was statistically significant $(F=5.65, \mathrm{df}=4 / 20, \mathrm{p}<.01$ ). A Student-NewmanKeuls range test performed on the mean number of correct responses in each day suggested that there

Table 1. Mean Proportion of Correct Responses in Successive Blocks of 25 Presentations in Each of 5 Successive Days

\begin{tabular}{|c|c|c|c|c|c|c|}
\hline Day & 1 & 2 & 3 & 4 & 5 & Mean \\
\hline Block 1 & .447 & .567 & .567 & .587 & .627 & .558 \\
\hline 2 & .460 & .487 & .567 & .640 & .620 & .555 \\
\hline 3 & .467 & .440 & .573 & .613 & .600 & .546 \\
\hline 4 & .533 & .507 & .640 & .667 & .693 & .608 \\
\hline 5 & .493 & .513 & .600 & .667 & .600 & .575 \\
\hline 6 & .447 & .433 & .627 & .587 & .600 & .546 \\
\hline 7 & .560 & .367 & .627 & .620 & .680 & .571 \\
\hline 8 & .573 & .480 & .600 & .700 & .653 & .601 \\
\hline Mean & .498 & .474 & .600 & .635 & .634 & \\
\hline
\end{tabular}

Table 2. Mean Proportion of Correct Responses as a Function of Lag

\begin{tabular}{rccccccrrr}
$\operatorname{Lag}$ & 0 & 2 & 4 & 6 & 8 & 10 & 12 & 14 & 16 \\
\hline $\mathrm{p}(\mathrm{c})$ & .987 & .742 & .669 & .578 & .556 & .509 & .532 & .531 & .501 \\
\hline
\end{tabular}

were no significant differences among Days 3,4 , and 5 and there was no difference between Days 1 and 2. However, the difference between Day 1 and Day 3 was significant $\left(W_{2}=2.38 ; p<.05\right)$, as was the difference between Day 2 and Day $3\left(W_{3}=2.89 ; p<.05\right)$. The results suggest that two practice sessions were necessary to stabilize Ss' behavior.

A second index suggested that Ss' ability to attend to each item improved with practice. The mean proportion of errors given a lag of zero was $\mathbf{0 4 5}$ on Day 1 and .128 on Day 2. In contrast, the mean proportion of errors given lag zero was $.019, .008$, and .008 for Days 3, 4, and 5, respectively.

Table 2 presents the mean proportion of correct responses as a function of lag, averaged over Ss and Days 3,4 , and 5 . The proportion of correct responses is reduced approximately $25 \%$ by two intervening items. Forgetting appears to asymptote at about lag 10 .

\section{References}

Hinrichs, J. V. Short-term memory with a guessing technique. $J$. exp. Psychol., 1966, 71, 89-95.

Peterson, L. R., \& Peterson, M. J. Short-term retention of individual verbal items. J. exp. Psychol., 1959, 58, 193-198.

Shepard, R. N., \& Teghtsoonian, M. Retention of information under conditions approaching a steady state. J. exp. Psychol., 1961, $62,302-309$.

Waugh, N. C., \& Norman, D. A. Primary memory. Psychol. Rev., $1965,72,89-104$.

\section{Notes}

1. This research was supported by a grant from the Office of Research Development of the University of Connecticut Research Foundation. Suzanne Standish ran SS and aided in analyzing data. 2. R. Bjork and R. Miller of the Institute for Mathematical Studies in the Social Sciences, Stanford University, constructed the algorhythm and computer program used to generate item sequences. 